\title{
PERCEÇÃO, EXPRESSÃO E VALORIZAÇÃO DAS EMOÇÕES DAS CRIANÇAS NA EDUCAÇÃO PRÉ-ESCOLAR
}

\author{
Maria Regina Teixeira Ferreira Capelo' \\ John Miguel Costa Varela ${ }^{2}$ \\ Noemí Serrano-Díaz ${ }^{3}$
}

\section{RESUMO}

A educação emocional é fundamental para a adaptação pessoal e social do indivíduo. Assim sendo, buscou-se por meio desta pesquisa adaptar a prova para avaliar a capacidade de perceber, expressar e valorizar emoções das crianças portuguesas na educação pré-escolar. Após equivalência semântica, operacional, conceptual e de itens, a prova foi respondida por 374 crianças. Os dados obtidos foram contrastados com critérios externos avaliados pelas representações das 26 educadoras de infância. Verificou-se maior número de acertos em relação à alegria e à tristeza na subescala perceção e expressão das emoções básicas com a escola privada a apresentar maior rendimento; que a perceção e valorização das emoções básicas aumenta em função da idade; que o género feminino apresenta maior performance na subescala perceção e valorização das emoções básicas; e relações significativas positivas entre as subescalas em análise e os critérios externos - adaptação, controlo, rendimento e aceitação, e correlações significativas negativas entre as subescalas e a conflitividade. A prova, embora em fase desenvolvimental, contribui para medir a adaptação pessoal e social das crianças portuguesas na etapa pré-escolar

Palavras-chave: Perceção Emocional. Valorização Emocional. Avaliação da Perceção Emocional.

\footnotetext{
1 Doutorada na Área do conhecimento da Psicologia Social pela Universidade de Cádis, professora em funções técnico-pedagógicas na Direção Regional de Educação, RAM, Portugal. E-mail: m.regina.capelo@gmail.com

2 Mestre em Famílias e Sistemas Sociais pelo Instituto Superior Miguel Torga, professor em funções técnico-pedagógicas na Direção Regional de Educação, RAM, Portugal. E-mail: jmcvarela@gmail.com

3 Doutorada em Ciências Sociais e Jurídicas pela Universidade de Cádis, Docente no Departamento de didática da Universidade de Cádis, Espanha. E-mail: noemi.serrano@uca.es
} 


\title{
PERCEPTION, EXPRESSION AND VALUING OF CHILDREN'S EMOTIONS IN PRE- SCHOOL EDUCATION
}

\begin{abstract}
Emotional education is fundamental for the individual's personal and social adaptation. Objective to adapt a test to assess the capacity to perceive, express and value emotions of Portuguese children in pre-school education. After the semantic, operational, conceptual and items equivalence, the questionnaire was answered by 374 children. The data obtained were compared with external criteria validated by 26 childhood educators. More positive results were found in relation to happiness and sadness in the perception and expression of basic emotions (higher achievements were identified in private schools); the perception and valuing of basic emotions increases according to the child's age; the feminine gender presents greater performance in the perception and valuing subscale; and significant positive relationships between the subscales under review and the external criteria adaptation, control, achievement and acceptance, and significant negative correlations between subscales and conflict. The test, although at a developmental stage, contributes to measuring the personal and social adaptation of Portuguese children in preschool.
\end{abstract}

Keywords: Emotional Perception. Emotional Valuing. Assessment of Emotional Perception.

\section{PERCEPCIÓN, EXPRESIÓN Y VALORIZACIÓN DE LAS EMOCIONES DE LOS NIÑOS EM EDUCACIÓN INFANTIL}

\section{RESUMEN}

La educación emocional es fundamental para la adaptación personal y social del individuo. Objetivo adaptar la prueba para evaluar la capacidad de percibir, expresar y valorar las emociones de los niños portugueses en Educación Infantil. Después de la equivalencia semántica, operacional, conceptual y de ítems, el ensayo fue contestado por 374 niños. Los datos obtenidos fueron verificados con criterios externos validados por 26 educadores de la etapa. Se verificó un mayor número de resultados en relación a la alegría y tristeza en la percepción y expresión de las emociones básicas con un mayor rendimiento en la escuela privada; la percepción y valoración de las emociones básicas aumenta en función de la edad; el género femenino presenta mayor desempeño en la subescala de percepción y valoración; que el género femenino presenta mayor rendimiento en la subescala de percepción y valoración. La prueba, aunque en desarrollo, contribuye a medir la adaptación personal y social de los niños portugueses en la etapa preescolar.

Palabras clave: Percepción emocional. Evaluación emocional. Educación emocional en la infancia. 


\section{INTRODUÇÃO}

Domínio fundamental do desenvolvimento humano, as emoções têm suscitado interesse científico desde longa data (JESUS, LEMPKE, 2015). Associadas a fatores externos ao indivíduo e indutoras de determinadas respostas expressas nas suas reações intensas e rápidas (ATKINSON, ATKINSON, SMITH, 2002; BOCK, FURTADO, TEIXEIRA, 2008), a manifestação emocional é definida como uma reação intensa a um evento significativo para $O$ indivíduo, acompanhada por manifestações fisiológicas e psicológicas (FREITAS-MAGALHÃES, 2013), que inclui uma componente biológica, cognitiva e comportamental, interligada a sensações subjetivas de prazer ou de dor (MOORS, 2013). Isto é, exercem efeitos motivadores sobre o comportamento e influenciam a perceção, a aprendizagem e o desempenho.

Desde cedo é possível observar alguns sinais de emoções programadas biologicamente, como nojo, interesse, angústia e satisfação em bebés recém-nascidos (JESUS, LEMPKE, 2015). Dos dois aos sete meses, percebe-se a demostração de novas emoções básicas como tristeza, raiva, alegria, surpresa e medo, as quais são percebidas em todos os bebés saudáveis da mesma idade e em diversas culturas (SHAFFER, KIPP, 2012). Aos dois anos surgem as emoções autoconscientes e avaliativas, mais complexas, como vergonha, culpa, inveja e orgulho (SHAFFER, KIPP, 2012) fundamentais para a compreensão das regras ou padrões sociais para avaliar as suas atitudes, estando associadas ao desenvolvimento cognitivo. Aos três anos a criança é capaz de avaliar as próprias emoções e a partir dos cinco anos de vida, as crianças ostentam algum controlo emocional (JESUS, LEMPKE, 2015). O desenvolvimento emocional ocorre através das interações sociais e afetivas vivenciadas pela criança, concorre para o seu desenvolvimento cognitivo, sendo imprescindível que, família e educadores, promovam múltiplas oportunidades de se relacionar com diferentes pessoas e situações. 
Plutchik (1980) preconiza a existência de oito emoções básicas: alegria, confiança, medo, surpresa, tristeza, nojo, irritação e antecipação. O modelo circumplexo, psicoevolucionário, integrativo, das emoções, representado pela "roda das emoções de Pluchik" pode assumir um modelo curvo a duas ou a três dimensões (PLUTCHIK, KELLERMAN, 2013), sustenta que as oito emoções básicas são biologicamente primitivas, que combinadas formam emoções mais complexas e evoluíram a fim de incrementarem a sobrevivência.

As seis emoções básicas demonstráveis nas expressões faciais - alegria, tristeza, medo, surpresa, raiva e nojo, - são referidas por Ekman (2007) como inatas e universais, produto da história evolutiva da humanidade (SHERER, EKMAN, 2014) podendo, combinadas, dar origem a emoções secundárias, mais complexas (IZARD, 2010).

A teoria preconizada por Ekman (2007) foi refutada pelos construtivistas ao defenderem uma abordagem cultural das emoções socialmente construídas. Evidências científicas sustentam as duas perspetivas apesar do debate relativo à universalidade das emoções (HARMON-JONES, SCHMEICHEL, MENNITT, HARMON-JONES, 2011) e à classificação das mesmas (GENDRON, ROBERSON, VAN DER VYER, BARRET, 2014; JACK, GARROT, YU, CLADARA, SCHYNS, 2012; LINDQUIST, SIEGEL, QUIGLEY, BARRET, 2013).

Reconhecer emoções nos rostos é essencial para a interação humana (AGUIAR, SILVA, AGUIAR, TORRO-ALVES, SOUZA, 2016). Elas são parte essencial da vida racional, intervêm em todo o processo evolutivo associado à comunicação, à interação social, ao processamento da informação, à vinculação, ao desenvolvimento moral, entre outras variáveis, com influência fulcral na vida quotidiana e no processo de tomada de decisão do ser humano (JESUS, LEMPKE, 2015; LÓPEZ CASSÁ, 2005; LOPATOVSKA, ARAPAKIS, 2011).

As emoções influenciam fortemente o processo de aprendizagem e a sua compreensão no contexto educacional é relevante (JESUS, LEMPKE, 2015). O desenvolvimento das capacidades implícitas na perceção, expressão e valorização das emoções das crianças em idade pré-escolar 
dependem do nível de maturação biológica, estimulação, aprendizagem e contexto. Por isso, o desenvolvimento da inteligência emocional depende da combinação entre desenvolvimento cognitivo e socio-emocional (MESTRE, GUIL, MARTINEZ-CABAÑAS, LARRÁN, GONZÁLEZ, 2011).

A inteligência emocional inclui a habilidade de perceber e expressar emoções, usar a expressão para facilitar a atividade cognitiva, compreender as emoções e regular as emoções para o crescimento pessoal e emocional (MAYER, SALOVEY, 2007). É uma forma de interagir com o mundo tendo em conta os sentimentos, e engloba habilidades como o controlo dos impulsos, a autoconsciência, a motivação, o entusiasmo, a preservança, a empatia, a agilidade mental, configura ímpetos de carácter como a autodisciplina, a compaixão ou o altruísmo, indispensáveis para uma adequada e criativa adaptação social (GOLEMAN, 1995).

Análises multigrupo multinível com crianças em desvantagem social integradas em salas com educadoras de infância que estabelecem interações com mais qualidade a nível emocional e organizacional demonstraram competências de regulação emocional mais elevadas (CADIMA, FERREIRA, GUEDES, VIEIRA, LEAL, MATOS, 2016). Mestre et al (2011) defendem que implementar programas de educação emocional e inteligência emocional na etapa Pré-Escolar é uma boa ideia.

Uma forma válida de ajudar a avaliar os referidos programas consiste no desenvolvimento de instrumentos de medida, válidos e fiáveis (MESTRE et al, 2011), sendo notório a escassez de instrumentos destinados a avaliar a perceção, expressão e valorização das emoções das crianças em idade pré-escolar.

Verificou-se que os instrumentos tendentes a avaliar a inteligência emocional, segundo Aslan e Erkus (2008), Davies, Lane, Devvonport e Scott (2010), podem ser agrupados em medidas de auto-relato ou auto avaliação, Medidas multifontes ou métodos dos informantes ( $360^{\circ}$ tests) e medidas de desempenho ou aptidão.

- Medidas de auto-relato ou auto avaliação: 
Neste domínio destaca-se 0 inicial Trait Emotional Intelligence Questionnaire (TEIQUe) desenvolvido por Petrides (PETRIDES, 2009) deu lugar a novas versões, designadamente, uma versão curta (TEIQUe-SF) (COOPER, PETRIDES, 2010; PETRIDES, 2011), uma versão para adolescentes (TEIQue-CE) (PETRIDES, VERNON, SCHAMER, VESELKA, 2011) e uma versão para crianças (TEIQUe-CF) (RUSSO, MANCINI, TROMBINI, BALDARO, MAVROVELI, PETRIDES, 2012). Questionário complementar ao TEIQue (TEIQue 360\%) é respondido por pessoas que têm contacto regular com o indivíduo que responde à prova de autorrelato. Quanto ao The Emotional Quotient Inventary (EQ-i) (VAN ZYL, 2014), posteriormente, foi construída uma versão reduzida (EQ-i:S) e outra para crianças e adolescentes (EQ-i:YV). A informação é obtida através do próprio sujeito sobre comportamentos que envolvem componentes emocionais. Segundo Davies e colaboradores (2010), apresentam o inconveniente de se basearem nas representações do indivíduo, produzindo uma avaliação do auto-conceito e não do constructo em si, mas, em contrapartida, tem a vantagem de ser de fácil aplicação e permite aceder às percepções do sujeito que não poderiam ser obtidas de outra forma.

- Medidas multifontes ou métodos dos informantes (360 tests):

The Emotional Quotient $360^{\circ}$ (EQ 360 ) completa o EQ-i e por norma é utilizado em contexto laboral e preenchido por colegas, chefes e/ou subordinados, mas também pode ser preenchido por familiares ou pessoas próximas do trabalhador (PETRIDES, FURNHAM, 2000). Também The Emotional and Social Intelligence Competency Inventory (ESCI-360) pretende medir a habilidade para perceber e aplicar o conhecimento emocional na relação com o outro (BAGUN, ISWARI, 2015). As medidas multifontes apoiam-se no autorrelato, mas distinguem-se pela utilização de múltiplas fontes. Este método permite medir a forma como o sujeito é percecionado pelos outros, mas não a verdadeira competência emocional (FIORI, ANTONAKIS, 2011).

- Medidas de desempenho ou aptidão:

Construído por Mayer, Salovey e Caruso, o Mayer-Salovey-Caruso Emotional Intelligence Tes (MSCEIT V2.0), apoia-se no modelo teórico de Salovey e Mayer, sendo de destacar que a área experiencial é avaliada 
através da percepção emocional e da facilitação do pensamento e a área estratégica pela compreensão e regulação emocional (RIVERS, BRACKETT, REYES, MAYER, CARUSO, SALOVEY, 2012). Esta prova possui uma versão delineada para pré-adolescentes e adolescentes intitulada Mayer-SaloveyCaruso Emotional Intelligence Test: Youth Version (MSCEIT-YV). As medidas de desempenho inserem-se nos modelos de habilidades ou competências e objetivam caracterizar o grau de desempenho objetivo no cumprimento ou resolução de determinada tarefa. Críticas têm envolvido a discussão à volta da subjetividade relacionada com a experiência emocional, contrariamente àquilo que acontece com os habituis testes de habilidades cognitivas (FIORI, ANTONAKIS, 2011).

A partir duma meta-análise sobre instrumentos de avaliação das habilidades emocionais das crianças, Humphrey, Kalambouka, Wigelsworth, Lendrum, Deighton, Wolpert (2011) identificaram doze medidas com características diferentes, sendo de destacar o Izard's Emotional Knowledge Test (EKT) de Izard, Fine, Schultz, Mostow, Ackerman \& Youngstrom (2001), baseado na compreensão e percepção das emoções; o Diagnostic Analysis of Nonverbal Accuracy (DANVA) de Nowicki e Duke (1994), assente no reconhecimento de rostos e da linguagem não-verbal em crianças a partir dos 4 anos; e o Assessment of Children's Emotional Skills (ACES) de Schultz, Izard, e Bear (2004) para avaliar a atribuição emocional nomeadamente, a felicidade, a tristeza e a raiva, em crianças no escalão etário 4 - 8 anos.

Pelo acima descrito, apraz-nos dizer que, a temática "emoções" tem ganho importância fulcral no campo educacional, no contexto escolar. Além disso, é imprescindível a existência de instrumentos que permitam medir e avaliar a eficácia dos programas de intervenção no âmbito da inteligência emocional na etapa pré-escolar (MESTRE et al, 2011).

Este estudo é o resultado da adaptação da prova para avaliar a capacidade de perceber, expressar e valorizar emoções das crianças portuguesas na educação pré-escolar. Especificamente: avaliar a capacidade da criança na educação pré-escolar de perceber e valorizar as emoções básicas; descrever os resultados da prova em função da idade, 
género e tipo de escola (pública/privada); e determinar as relações entre as representações de juízes externos (educadoras de infância das crianças) e as subescalas e a pontuação total da prova.

\section{MÉTODO}

\section{Design}

Assente numa abordagem quantitativa, descritiva, correlacional e de corte transversal.

\section{Participantes}

Participaram 374 crianças da educação pré-escolar (47,60\% meninas e $52,40 \%$ meninos), com idades compreendidas entre 3 e 6 anos ( 3 anos= $34,80 \% ; 4$ anos $=31,60 \% ; 5$ anos $=25,90 \%$ e 6 anos $=7,80 \% ; M=4,07$ e $D P=0,96$ ). Os jardins de infância ou unidades de educação pré-escolar têm diferentes idiossincrasias: 5 privados (78,87\% participantes), situados em meio urbano e 2 públicos (21,13\% participantes), um em meio urbano e um em meio rural, sendo as crianças dos estabelecimentos privados provenientes de um nível socioeconómico mais elevado do que o público. A amostra contou com a participação de 26 educadoras de infância docentes das crianças envolvidas no estudo.

\section{Instrumento}

A prova para avaliar a capacidade de perceber, expressar e valorizar emoções das crianças na etapa pré-escolar, disponibilizada por Jose Miguel Mestre do Laboratório de Inteligencia Emocional da Universidade de Cádis, na versão inicial em espanhol, foi desenvolvida por Mestre, Guil, MartinezCabañas, Larrán e González (2011). Apoiada no modelo de Mayer e Salovey (2007) é uma prova construída em PowerPoint para ser apresentada às crianças (entre 3 e 6 anos) no écran do computador. $O$ aplicador pede às crianças que respondam às perguntas assinalando no ecrã apenas a resposta que consideram correta de acordo com as instruções relativas a cada diapositivo. Os 16 itens da prova são: 
1. Assinala a cara que Alex apresentaria se estivesse triste porque 0 professor/a brigou com ele na aula?;

2. Qual das jovens sente raiva porque the disseram algo que não agradou? Cris, Tina ou nenhuma das duas?;

3. Alex estava dormindo e acordou com um ruído forte. Estava às escuras no quarto e sentia medo. Que cara achas que ele apresentou?;

4. Qual das duas achas que está mais triste? Cris, Tina ou nenhuma das duas?;

5. Alex está com raiva porque o amigo tem um jogo novo, mas não o quer emprestar. Que cara achas que apresentou?;

6. Qual das duas achas que está mais triste? Cris, Tina ou nenhuma das duas?;

7. Que cara achas que Alex mostrava se se sentisse só e triste porque ninguém queria brincar com ele no recreio?;

8. Qual das duas achas que está com muito medo? Cris, Tina ou nenhuma delas?;

9. Alex está com raiva porque o seu irmão mais velho estragou o seu jogo preferido. Que cara achas que ele mostrou?;

10. Qual das duas achas que está muito surpreendida? Cris, Tina ou nenhuma das duas?;

11. Alex está alegre com a sua festa de aniversário. Que cara achas que terá?;

12. Qual das duas achas que sente nojo? Cris, Tina ou nenhuma das duas?;

13. Alex chega a casa e encontra uma festa que não esperava, organizada pela mãe, e está um pouco surpreendido. Que cara achas que ele apresenta?;

14. Qual das duas achas que está mais alegre? Cris, Tina ou nenhuma das duas?;

15. Hoje ofereceram ao almoço uma comida que Alex detestava e sentiu nojo. Que cara achas que mostrou?;

16. Qual das duas está menos triste? Cris, Tina ou nenhuma das duas? 
Dos 16 itens apresentados, oito correspondem à subescala de emoções básicas sendo solicitado às crianças que assinalem entre seis hipóteses (seis caras representando diferentes expressões faciais) em cada diapositivo, qual expressão teria a personagem (Alex) em determinada situação (itens: $1,3,5,7,9,11,13,15$ ) conforme exemplificado na figura 1 . Isto é, a perceção da emoção corresponde à expressão facial que a personagem estaria a adotar numa situação concreta. A fiabilidade interjuízes (12 especialistas em emoções), correlações intraclasses, para os itens desta subescala, foi de 0.93 (MESTRE et al, 2011).

FIGURA 1 - Diapositivo para avaliação da capacidade da criança para perceber, valorizar e expressar as emoções básicas
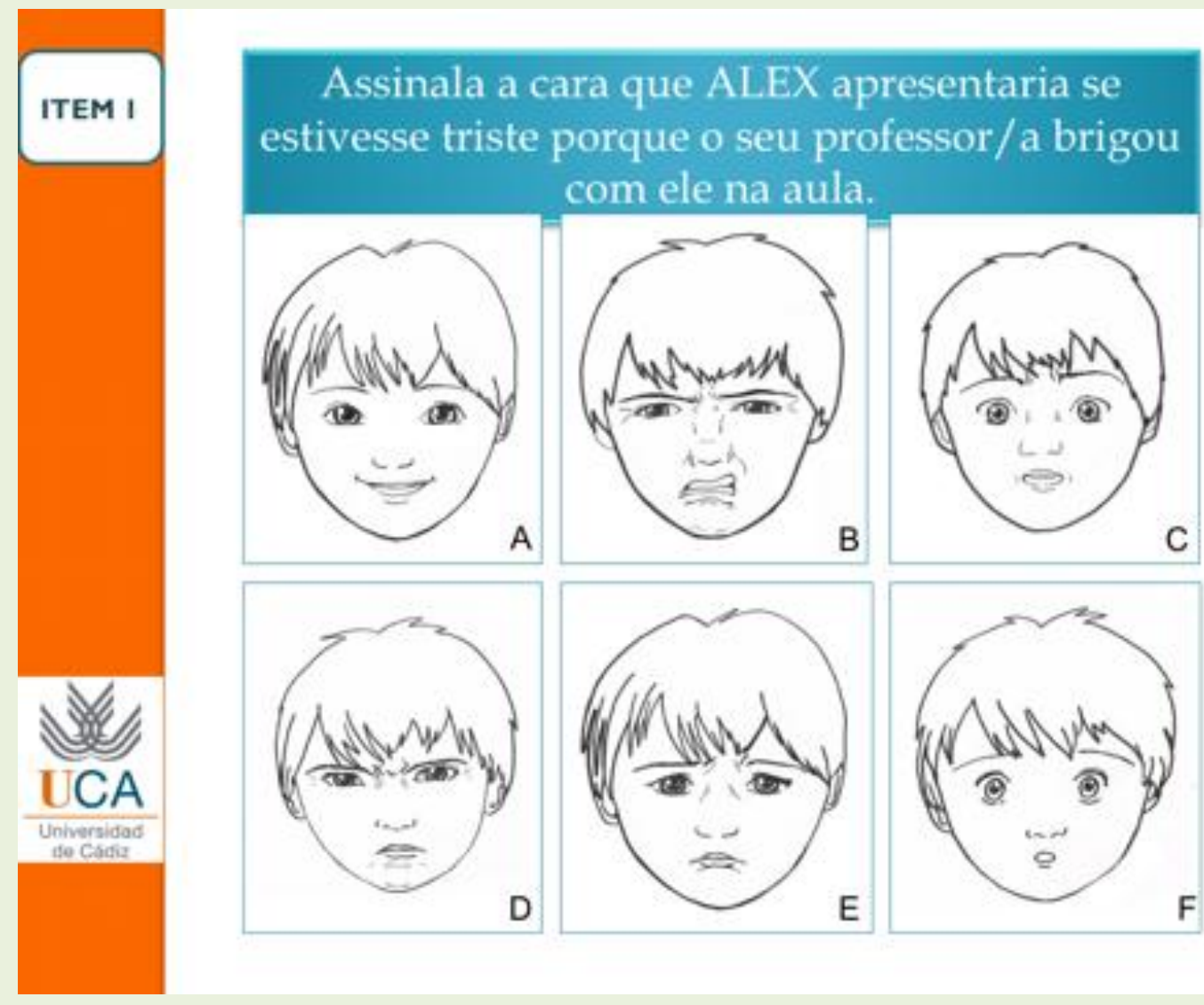

Fonte: Mestre et al, 2011.

Os restantes oito itens procuram avaliar situações mais ambíguas para a criança, que num elenco de três respostas, tem de discriminar entre duas personagens idênticas (irmãs gémeas: Cris e Tina ou nenhuma das duas), qual das duas provavelmente está a sentir uma emoção ou nenhuma das 
duas (itens: $2,4,6,8,10,12,14,16$ ). Esta situação requer a perceção e depois a valorização e esforço da criança para discernir a resposta mais ajustada à questão colocada. A correlação intraclasse de 12 especialistas em emoções foi de 0,90 para os itens desta subescala (MESTRE et al, 2011). Os itens desenhados geram dois tipos de variáveis independentes: valorização de emoções; perceção e valorização de expressões faciais (figura 2). A correlação intraclasse de 12 especialistas em emoções foi de 0,90 para os itens desta subescala (MESTRE et al, 2011).

FIGURA 2 - Diapositivo para avaliação da capacidade da criança para perceber e considerar qual das personagens se ajusta melhor ao conjunto de três respostas: Cris, Tina ou nenhuma das duas

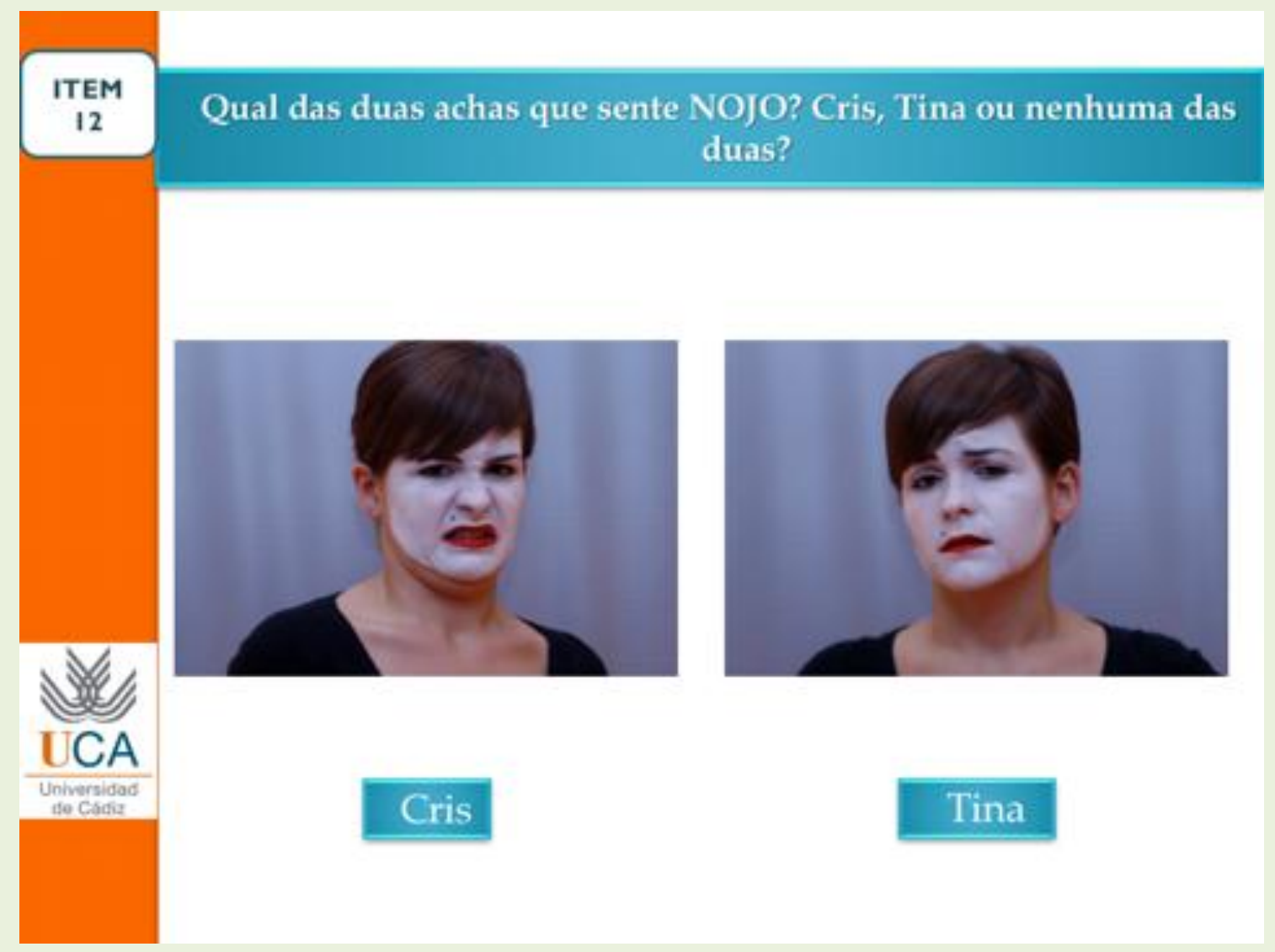

Fonte: Mestre et al, 2011.

As respostas nunca apareciam pela mesma ordem e incluíam etiquetas emocionais - alegria, tristeza, medo, raiva, surpresa e nojo. A aplicação da prova não tem tempo limite determinado e as respostas são 
anotadas na folha de observação do avaliador, na qual consta o nome, idade, género e escola da criança.

Além da prova aplicada às crianças é solicitado às respetivas educadoras de infância o preenchimento de um questionário em forma de escala de Likert de seis pontos (1 corresponde a Nada ou Quase Nada, 2 Um Pouco, 3 Médio, 4 Bastante, 5 Muito e 6 corresponde a Completamente), com 5 questões que visam apreender as representações sobre o nível de adaptação da criança (Mestre et al, 2011). As questões foram:

1) Qual é o nível de adaptação da criança à escola? Com esta questão procurava-se conhecer o nível de integração dos alunos à escola, se gostava de participar, se cumpre as normas estabelecidas na aula, se realiza as tarefas ou atividades solicitadas.

2) Qual é o nível de controlo dos impulsos da criança? Impulsividade entendida como atuar sem refletir, resposta muito rápida e impaciência da criança; baixo controlo dos impulsos e pouca tolerância à frustração.

3) Qual é o nível de rendimento académico da criança? Entendido como uma medida do desenvolvimento do aluno na respectiva etapa ou nível.

4) Qual é o nível de aceitação desta criança pelos pares? Se a criança é bem aceita pelo grupo ou turma, se interage com eles, se apresenta caraterísticas de liderança, se é requerido ou seguido pelos outros.

5) Qual é o nível de conflictividade da criança? Se é agressivo (verbal ou fisicamente), se tem problemas em cumprir as regras na aula ou no recreio, se respeita colegas e docentes.

\section{Procedimentos}

Procurando alcançar a maior equivalência possível entre o instrumento aplicado em línguas diferentes realizou-se um processo de tradução e versão (forward and backtranslation) (HERDMAN, FOX-RUSHBY, BADIA, 1997). 
Três profissionais com experiência em emoções e fluentes no espanhol realizaram traduções independentes. Estas foram comparadas e discutidas, concluindo-se uma primeira versão da prova. Outra tradução foi realizada por um professor de espanhol, fluente em português. Cada item foi comparado com o original espanhol, apreciando-se a equivalência semântica e o sentido geral do item. Essa versão foi traduzida para o espanhol (backtranslation) por um professor de nacionalidade espanhola e com fluência nos dois idiomas. Foi então enviada ao professor Jose Miguel Mestre, um dos construtores da escala, que anviu a semântica utilizada.

Após autorização da Secretaria Regional de Educação bem como dos Jardins de Infância e Unidades de Educação Pré-escolar, para aplicação da prova, procedeu-se ao pedido de anuência dos encarregados de educação e das educadoras de infância das crianças. Foram cumpridos os procedimentos éticos nomeadamente o consentimento livre e informado bem como a confidencialidade e o anonimato (FELDMAN, 2001). Cada aplicador assegura-se que a criança compreendeu as questões e se não o fazia solicitava que assinalasse a imagem da emoção descrita no écran.

A reação observada pelos diversos aplicadores da prova PERCEVAL V2.0., perante a aplicação da mesma, foi considerada muito positiva tanto pelas crianças como pelas educadoras de infância. A duração da prova variou entre 10 a 15 minutos.

Os dados obtidos foram inseridos e analisados estatisticamente no software Special Package for Social Sciences (SPSS), versão 23 para 0 Windows (MARTINS, 2011). Face à dimensão da amostra ( $N=374)$, os testes estatísticos elegidos são os paramétricos (FORTIN, 2009; MARTINS, 2011) porque permitem examinar diferenças ou relações significativas entre variáveis intervalares.

\section{RESULTADOS}

O objetivo inicial consiste em avaliar a capacidade de a criança em idade pré-escolar perceber e valorizar as emoções básicas (tabela 1). Os resultados indicam que as crianças em idade pré-escolar têm mais 
facilidade para perceber e identificar emoções como a alegria $(70,90 \%)$ e a tristeza $(67,90 \%)$, menos acertos no reconhecimento de emoções como a raiva $(17,90 \%$ e $23 \%)$ e o medo $(16,60 \%)$ e confusão relativamente a emoções como a raiva $(17,9 \%$ e $23,0 \%)$ e o nojo $(71,4 \%$ e $63,4 \%)$, e o medo $(16,6 \%)$ e a surpresa $(41,4 \%)$. Assim, a tabela 1 mostra a percentagem de respostas corretas de cada item na subescala "perceção de emoções básicas".

TABELA 1 - Percentagem (\%) de acertos de cada item por resposta dada numa amostra de 374 crianças de 3 a 6 anos na subescala perceção de emoções básicas

\begin{tabular}{cccccccc}
\hline & NS/NR & Alegria & Nojo & Medo & Raiva & Tristeza & Surpresa \\
\hline Item 1 & 0,5 & 1,1 & 15,0 & 7,5 & 3,7 & $67,9^{*}$ & 4,3 \\
Item 3 & 0,3 & 1,3 & 19,0 & $16,6^{*}$ & 8,0 & 13,4 & 41,4 \\
Item 5 & 0,3 & 2,9 & 71,4 & 1,6 & $17,9^{*}$ & 4,8 & 1,1 \\
Item 7 & 0,3 & 1,1 & 10,7 & 5,3 & 8,3 & $67,9^{*}$ & 6,4 \\
Item 9 & 0 & 1,6 & 63,4 & 1,9 & $23,0^{*}$ & 8,6 & 1,6 \\
Item 11 & 0 & $70,9^{*}$ & 4,3 & 8,6 & 4,3 & 4,5 & 7,5 \\
Item 13 & 0,3 & 29,4 & 11,5 & 15,0 & 7,0 & 7,8 & $29,1^{*}$ \\
Item 15 & 0 & 3,7 & $57,2^{*}$ & 7,5 & 13,9 & 12,0 & 5,6 \\
\hline
\end{tabular}

Fonte: elaboração própria

TABELA 2 - Percentagem (\%) de acertos de cada item por resposta dada numa amostra de 374 crianças de 3 a 6 anos na subescala valorização das emoções

\begin{tabular}{ccccc}
\hline & NS/NR & Cris & Tina & Nenhuma \\
\hline Item 2 & 1,1 & 36,9 & $54,5^{*}$ & 7,5 \\
Item 4 & 0,3 & 74,9 & 8,8 & $16,0^{*}$ \\
Item 6 & 0 & $91,7^{*}$ & 7,2 & 1,1 \\
Item 8 & 0 & 4,3 & $94,7^{*}$ & 1,1 \\
Item 10 & 0,3 & 35,8 & 51,1 & $12,8^{*}$
\end{tabular}




\begin{tabular}{ccccc} 
Item 12 & 0 & $90,6^{*}$ & 8,0 & 1,3 \\
Item 14 & 0 & 10,4 & $88,5^{*}$ & 1,1 \\
Item 16 & 0 & 50,3 & $35,3^{*}$ & 14,4 \\
\hline
\end{tabular}

Fonte: elaboração própria

Pela observação da tabela 2 se depreende que a valorização das emoções obriga a um maior esforço cognitivo da criança uma vez que tem de comparar duas figuras com a possibilidade de que nenhuma corresponda às gémeas do item (item 10). As crianças apresentam maior facilidade quando a resposta correta corresponde a uma das personagens (Cris ou Tina) e o número de acertos é manifestamente menor quando a possibilidade que se ajusta à questão colocada é "nenhuma das duas".

No que concerne à primeira subescala "perceção de emoções básicas", podemos verificar a média foi de 44,12 (M=44,12; $D P=19,60)$; a percentagem mínima foi 0 e a máximo foi 88. Em relação à segunda subescala "perceção e valorização de emoções básicas", podemos observar a média foi de 60,66 (M=60,66; $D P=14,33)$, apresentando um valor mínimo de 13 e um valor máximo de 100. Quanto à total pontuação, verificase uma média de 52,28 ( $M=52,28 ; D P=13,43)$, tendo a percentagem mínima 13 e a percentagem máxima 88.

A subescala "perceção e valorização de emoções básicas" obteve a média de respostas corretas mais elevada $(M=60,66 ; D P=14,33)$, seguida da escala total $(M=52,28 ; D P=13,43)$ e por fim, a subescala perceção e expressão de emoções básicas ( $M=44,12 ; D P=19,60)$ (Tabela 3).

TABELA 3 - Medidas de tendência central e dispersão referentes às respostas dadas pelas crianças

\begin{tabular}{cccc}
\hline & $\begin{array}{c}\text { Subescala } \\
\text { Perceção de emoções } \\
\text { básicas }\end{array}$ & $\begin{array}{c}\text { Subescala Perceção e } \\
\text { Valorização de emoções } \\
\text { básicas }\end{array}$ & $\begin{array}{c}\text { Total } \\
\text { Pontuação }\end{array}$ \\
\hline Média & 44,12 & 60,66 & 52,28
\end{tabular}


Desvio Padrão

Mínimo

Máximo
19,60

0

88
14,33

13

100
13,43

13

88

Fonte: elaboração própria

O objetivo seguinte visa obter a análise dos dados em função da idade, género e tipo de escola (pública/privada).

Verificou-se que existem diferenças estatisticamente significativas nas três escalas, em função da idade. A análise de variância One way ANOVA e Post-hoc de Bonferroni para comparações múltiplas (MARTINS, 2011) indica diferenças significativas nas três escalas, em função da idade. Os dados sugerem que as crianças aumentam progressivamente a sua precisão em perceber, expressar e valorizar emoções à medida que a idade aumenta. As crianças com três anos apresentam o resultado mais baixo ( $M=33,37$; $D P=$ $18,44)$ e as que têm seis anos responderam de forma mais correta $(M=57,34$; $\mathrm{DP}=15,83)$ na subescala perceção e expressão de emoções básicas $[F(3,370)=29,50 ; p=0,000)]$. Quanto à subescala perceção e valorização de emoções básicas nota-se que as crianças com três anos revelam o valor mais baixo $(M=56,63 ; D P=14,17)$, e as crianças com cinco anos de idade expressam maior perceção e valorização de emoções $(M=65,97 ; D P=13,32)$, $[F(3,370)=8,50 ; p=0,000)]$. Relativamente à pontuação total da escala, verifica-se diferenças em termos etários entre as crianças com três anos $(M=$ $44,90 ; D P=12,16)$ e as crianças com seis anos de idade $(M=61,66 ; D P=10,40)$; observa-se diferenças significativas entre crianças com quatro anos $(M=$ $52,78 ; D P=12,48)$ e as crianças com seis anos $(M=61,66 ; D P=10,40)[F(3,370)=$ $31,60 ; p=0,000)]$.

Para saber em que medida o género pode influenciar as perceções das emoções propostas pela prova, realizou-se o teste de diferenças entre as médias dos grupos para amostras independentes, o teste t-Student (FORTIN, 2009; MARTINS, 2011). A análise de amostras independentes (género) para cada subescala confirmou a influência da variável género. Os resultados indicam que o género feminino possui pontuações mais elevadas nas 
escalas utilizadas. No concernente à subescala perceção e expressão de emoções básicas, o género feminino apresenta nível mais elevado ( $M=$ $44,65 ; D P=20,40 ; p>0,050)$ do que o género masculino $(M=43,64 ; D P=18,88$; $p>0,050)$. Em relação à subescala perceção e valorização de emoções básicas verificamos também que as raparigas obtiveram pontuação estatisticamente significativa ( $M=62,41$; $D P=13,95)$ em relação aos rapazes $(M=59,07 ; D P=14,52)$, pois, $t(372)=-2,26, p=0,024$. E por fim, na pontuação total observamos que as meninas possuem melhores resultados ( $M=53,48$; $D P=13,90 ; p>0,050$ ) enquanto os meninos obtiveram um valor mais baixo $(M=51,18 ; D P=12,92 ; p>0,050)$.

No que concerne às diferenças entre escola pública ou privada, o teste t-Student, mostrou que há diferenças estatisticamente significativas na subescala perceção e expressão de emoções básicas, com as crianças do ensino privado a apresentarem melhor rendimento $(M=45,53 ; D P=19,33)$ que as do ensino público $(M=38,86 ; D P=19,83)$ e $+(372)=2,70 ; p=0,007$. Os dados não mostram diferenças significativas na subescala escala valorização das emoções e na escala total $(p>0,05)$. O número de alunos envolvidos no presente estudo difere entre o público $(N=79)$ e o privado $(N=295)$, mas por isso, neste universo, torna-se difícil determinar se o fator tipo de escola é uma variável importante no desenvolvimento das capacidades para perceber, expressar e valorizar emoções básicas.

De acordo com os critérios previamente estabelecidos, o último objetivo consiste em determinar as relações entre as representações de juízes externos (educadoras de infância das crianças) e as subescalas e a pontuação total da prova. Trata-se de medir a influência que uma variável exerce sobre uma ou mais variáveis e determinar em que medida esta explica o valor destas últimas (FORTIN, 2009). A tabela 4 mostra as medidas de tendência central e dispersão referentes às respostas dadas pelas educadoras de infância sobre a adaptação pessoal e social das crianças.

$O$ valor mais elevado incide na aceitação da criança pelos pares $(M=$ 5,17; $D P=0,95)$, seguindo-se a adaptação $(M=5,15 ; D P=1,07)$, o rendimento 
académico $(M=4,60 ; D P=1,08)$, o controlo dos impulsos $(M=4,09 ; D P=1,20)$ e por fim, a pontuar mais baixo a conflitividade $(M=2,50 ; D P=1,30)$.

TABELA 4 - Medidas de tendência central e dispersão referentes às respostas dadas pelas educadoras de infância acerca da adaptação pessoal e social das crianças

\begin{tabular}{lcccc}
\hline & Média & Desvio Padrão & Mínimo & Máximo \\
\hline Adaptação & 5,15 & 1,07 & 1,00 & 6,00 \\
Controlo & 4,09 & 1,20 & 1,00 & 6,00 \\
Rendimento & 4,60 & 1,08 & 1,00 & 6,00 \\
Aceitação & 5,17 & 0,95 & 2,00 & 6,00 \\
Conflitividade & 2,50 & 1,30 & 1,00 & 6,00 \\
\hline
\end{tabular}

Fonte: elaboração própria

O coeficiente de correlações $r$ de Pearson é utilizado para verificar a relação linear entre as variáveis critério (FORTIN, 2009; MARTINS, 2011) (as representações das educadoras de infância sobre os participantes) e as subescalas e a pontuação total da prova (tabela 5). Os resultados sugerem que a subescala perceção e expressão de emoções básicas se correlaciona significativamente com todas as variáveis critério, sendo positiva com a adaptação $(r=0,162)$, controlo $(r=0,138)$, rendimento $(r=0,317)$ e aceitação $(r=0,163)$ e negativa com a conflitividade $(r=-0,242)$. A subescala perceção e valorização de emoções básicas correlaciona-se significativa e positivamente com a adaptação $(r=1,77)$, controlo $(r=0,111)$, rendimento $(r=$ $0,189)$ e aceitação $(r=0,163)$. A pontuação da prova total apresenta associação significativa e positiva com a adaptação $(r=0,214)$, controlo ( $r=$ $0,162)$, rendimento $(r=0,335)$ e aceitação $(r=0,204)$, e negativa com a conflitividade $(r=-0,228)$. Isto é, a perceção e valorização das expressões emocionais primárias relacionam-se significativa e positivamente com as representações das educadoras de infância sobre o nível de adaptação do aluno à escola, o nível de controlo dos impulsos, o rendimento académico e 
a aceitação pelos pares. O coeficiente de correlação negativa significa que quanto maior for o nível de perceção, expressão e valorização das emoções, menor é o nível de conflitividade na idade pré-escolar.

TABELA 5 - Matriz de correlações entre as subescalas da prova e pontuação total da prova e os critérios estabelecidos

\begin{tabular}{lccc}
\hline & $\begin{array}{c}\text { Perceção emoções } \\
\text { básicas }\end{array}$ & $\begin{array}{c}\text { Perceção e valorização de } \\
\text { emoções básicas }\end{array}$ & $\begin{array}{c}\text { Pontuação } \\
\text { Total }\end{array}$ \\
\hline Adaptação & $0,162^{* *}$ & $0,177^{* *}$ & $0,214^{* *}$ \\
Controlo & $0,138^{* *}$ & $0,111^{*}$ & $0,162^{* *}$ \\
Rendimento & $0,317^{* *}$ & $0,189^{* *}$ & $0,335^{* *}$ \\
Aceitação & $0,163^{* *}$ & $0,163^{* *}$ & $0,204^{* *}$ \\
Conflitividade & $-0,242^{* *}$ & $-0,093$ & $-0,228^{* *}$ \\
\hline
\end{tabular}

*A correlação é significativa no nível 0,05.

**A correlação é significativa no nível 00,1.

Fonte: elaboração própria

\section{DISCUSSÃO E CONCLUSÕES}

O presente estudo cumpre com o seu objetivo, ao disponibilizar a primeira versão da prova para "Avaliar a capacidade de perceber, expressar e valorizar emoções das crianças na educação pré-escolar", em português. Este instrumento, apoiado no modelo de inteligência emocional de Mayer e Salovey (2007), desenvolvido por Mestre e colaboradores (2011), procura avaliar a capacidade da criança e não a perceção da sua capacidade. Estabelece-se, assim, uma contribuição para tornar possível o diagnóstico precoce da capacidade de perceber, expressar e valorizar emoções das crianças portuguesas em etapa educação pré-escolar.

Ainda não existe consenso de como operacionalizar a tradução de uma escala. Procurou-se a equivalência conceptual e de itens com o intuito de verificar no novo contexto a pertinência dos domínios abrangidos pela prova inicial. A equivalência semântica tem importância fulcral, pois espelha 
a correspondência de sentidos dos constructos entre o instrumento original e a versão traduzida, esperando-se que propicie efeito semelhante na população em diferentes culturas, conforme preconiza Ekman (2007). No entanto, Harmon-Jones, Schmeichel, Mennitt \& Harmon-Jones (2011) questionam a universalidade das emoções. Não há dúvidas, porém, que perceber as emoções expressas no rosto é indispensável na interação humana (AGUIAR, SILVA, AGUIAR, TORRO-ALVES, SOUZA, 2016; JESUS, LEMPKE, 2015; LÓPEZ CASSÁ, 2005; LOPATOVSKA, ARAPAKIS, 2011). Contudo, fatores socioculturais estão presentes nos indivíduos e emergem de um conjunto heterogéneo de variáveis que incluem a linguagem, a etnia, a religião, as crenças, valores, relações interpessoais, organização social, entre outros (JORGE, 1998). Torna-se, por isso evidente que, na tradução da escala, seja tido em conta a adaptação transcultural.

A tradução e back-translation (HERDMAN, FOX-RUSHBY, BADIA, 1997) realizadas por profissionais bilingues especialistas em emoções permitiram recolher algumas imprecisões subtis; feitas as alterações obteve-se uma tradução literal. A versão final mostrou-se equivalente operacionalmente, uma vez que manteve o nome das personagens (Alex, Cris e Tina), respeitou o formato das questões e o veículo utilizado (PPT no écran do computador para as crianças e papel para as educadoras de infância), não recebeu críticas durante a aplicação, atendeu às expectativas dos investigadores e mereceu grande recetividade por parte das crianças. A aplicação individual da prova demonstrou-se morosa, porém não era possível de outro modo, pois a maioria das crianças não dispunha da competência leitora e era necessário assegurar que a compreensão da questão tinha sido alcançada.

Após aplicação da prova para avaliar a capacidade da criança na educação pré-escolar de perceber e valorizar as emoções básicas, em dois estabelecimentos de educação pré-escolar públicos e cinco jardins de infância privados, verificou-se que, tal como no estudo de Mestre et al (2011), as pontuações mais elevadas incidiram na alegria e na tristeza, notando-se dificuldade na perceção das restantes emoções básicas conforme refere a literatura (AGUIAR et al, 2016; MACHADO, VERÍSSIMO, 
TORRES, PECEGUINA, SANTOS, ROLÃO, 2008). Neste estudo, as crianças apresentaram melhor desempenho na subescala perceção e valorização das emoções básicas apesar de esta tarefa obrigar a um maior esforço cognitivo. Estes resultados contrariam os de Mestre e colaboradores (2011) uma vez que a pontuação mais elevada corresponde à subescala perceção das emoções básicas. A concorrer para o resultado obtido poderá influenciar a implementação de programas de educação emocional que potenciem a capacidade de optar corretamente em situações ambíguas. Esta situação requer novos estudos que confluam para um esclarecimento cabal dos resultados obtidos uma vez que amostra estudada incidiu maioritariamente em escolas privadas, num contexto insular.

Verificam-se diferenças estatisticamente significativas nas três escalas, em função da idade. Em conformidade com referenciais teóricos (FINE, IZARD, TRENTACOST, 2006; MESTRE et al, 2011; SAARNI, 2011) os resultados revelam que, à medida que a idade aumenta, as crianças incrementam progressivamente a sua precisão em perceber, expressar e valorizar emoções básicas.

A amostra sugere que as meninas têm mais facilidade em perceber eficazmente as emoções uma vez que pontuaram mais alto nas subescalas, sendo essa diferença estatisticamente significativa em relação à perceção e valorização das emoções. Neste âmbito, Halberstadt e Lozada (2011) realçam que as raparigas parecem apresentar uma maior capacidade para identificar corretamente emoções. Os resultados contrastam com os obtidos por Mestre e colaboradores (2011) uma vez que não encontraram diferenças estatisticamente significativas em função do género.

Este estudo confirma parcialmente a perspetiva de Ricarte, Minervino, Roazzi, Dias e Viana (2009). Isto é, crianças das escolas privadas pontuam mais alto no domínio das competências emocionais do que crianças das escolas públicas. Os resultados colocam as escolas privadas em primazia e sugerem diferenças intergrupais estatisticamente significativas, apenas na 
subescala perceção de emoções básicas. Neste domínio, a pesquisa de Mestre et al (2011), não foi conclusiva.

Os resultados assinalam relações significativas positivas entre os critérios externos relacionados com a adaptação pessoal e social da criança, designadamente a adaptação, o controlo, o rendimento e a aceitação e as subescalas em análise. Verificou-se também correlações significativas negativas entre as subescalas perceção de emoções básicas, perceção e valorização de emoções básicas e pontuação total e a conflitividade. Neste âmbito, a aceção das educadoras de infância participantes neste estudo confere com a literatura da especialidade, isto é, as crianças com maior perceção das emoções básicas manifestam melhor ajustamento social (IZARD, FINE, SCHULTZ, MOSTOW, ACKERMAN, YOUNGSTROM, 2001), mais sucesso nas interações sociais (MACHADO, VERÍSSIMO, DENHAM, 2012), apresentam maior adaptação ao contexto escolar, possuem mais controlo da impulsividade, obtêm melhor rendimento académico (IZARD et al, 2001), são menos conflituosas (MESTRE et al, 2011) e recorrem a estratégias adequadas para regularem as suas emoções (RHOADS, WARREN, DOMITROVICH, GEENBERG, 2011 ). Não foi possível avaliar se as informações prestadas pelas educadoras de infância sobre o nível de adaptação pessoal e social dos seus alunos foram afetadas por critérios de desejabilidade social, efeito pigmaleão, expetativas pessoais, entre outros fatores. Para colmatar esta limitação seria interessante obter a perspetiva dos pais/encarregados de educação, auxiliares de ação educativa que acompanham as crianças nos recreios e adicionar ao estudo outras variáveis como o envolvimento parental, o número de irmãos, meio urbano/rural. No entanto, vale salientar que as educadoras de infância, através da qualidade das interações que estabelecem, desempenham um importante papel moderador no desenvolvimento da regulação emocional das crianças em idade pré-escolar (CADIMA et al, 2016).

Por fim, após observados os aspetos relacionados com a equivalência semântica, operacional, conceptual e de itens, parece que o instrumento "prueba para evaluar la capacidade de percibir, expressar y valorar 
emociones em niños de la etapa infantil", adaptado para o português na sua versão inicial, requer uma posterior avaliação psicométrica para verificação da equivalência de mensuração. Contudo, estamos em crer que o mesmo contribui para medir a adaptação pessoal e social das crianças portuguesas na etapa pré-escolar.

\section{REFERÊNCIAS}

AGUIAR, J. S. R.; SILVA, A. I. P.; AGUIAR, C. S. R.; TORRO-ALVES, N.; SOUZA, W. C. A influência da intensidade emocional no reconhecimento de emoções em faces por crianças brasileiras. Universitas Psychologica, v. 15, n. 5, p. 206215, 2016.

ASLAN, S.; ERKUS, A. Measurement of emotional intelligence: validity and reliabillity studies of two scales. World Applied Sciences Journal, v. 4, n. 3, p. 430-438, 2008.

ATKINSON, R. L.; ATKINSON, R. C., SMITH, E. E.; BEM, D. J.; NOLEN-HOEKSEMA, S. Introdução à psicologia de Hilgard. 13. ${ }^{a}$ ed. Porto Alegre: Artmed, 2002.

BANGUN, Y., R.; ISWARIS. K. R. Searching for emotional intelligence measurement in Indobesia contexto with innovattive approach. ProcediaSocial and Behavioral Sciences, v. 169, p. 337-345, 2014.

BOCK, A. M. B.; FURTADO, O.; TEIXEIRA, M. L. T. Psicologias: uma introdução ao estudo da psicologia. 14. Ed. São Paulo: Saraiva, 2008.

CADIMA, J.; FERREIRA, T.; GUEDES, C.; VIEIRA, J.; LEAL, T.; MATOS, P. M. Risco e regulação emocional em idade pré-escolar: a qualidade das interações dos educadores de infância como potencial moderador. Análise Psicológica, v. 34, n. 3, p. 235-248, 2016.

CADIMA, J.; VERSCHUEREN, K.; LEAL, T.; GUEDES, C. Classroom interactions, dyadic teacher-child relationship, and self-regulation in socially disadvantaged young children. Journal of Abnormal Child Psychology, v. 44, p. 7-17, 2016.

COOPER, A.; PETRIDES, K. A psychometric analysis of the trait emotional quetionnaire-short form (TEIQUe-SF) using item response theory. Journal of Personality Assessment, v. 92, n. 5, p. 449-457, 2010.

DAVIES, K.; LANE, A.; DEVONPORT, T.; SCOTT, J. Validity and Reliability of a Brief Emotional Inteligence Scale (BEIS-10). Journal of Individual Differences, v. 31, n. 4, 2010.

EKMANk, P. Emotions Revealed: recognizing faces andfeelings to improve communication and emotional of findings. London: Elsevier, 2007.

FELDMAN, R. Compreender a Psicologia. Amadora: McGraw-Hill. 2001. 
FINE, S. E.; IZARD, C. E.; TRENTACOSTA, C. J. Emotion situation knowledge in elementary school: Models of longitudinal growth and preschool correlates. Social Development, v. 15, n. 4, p. 730-751, 2006.

FIORI, M.; ANTONAKIS, J. The ability modelo of emotional intelligence: Searching for valid measures. Personality and Individual Differences, v. 50, n. 3, p. 329-334, 2011.

FORTIN, M-F. Fundamentos e etapas do processo de investigação. Loures: Lusodidacta, 2009.

FREITAS-MAGALHÃES, A. A Psicologia das emoções: o fascínio do rosto humano. Lisboa. Leya, 2013.

GENDRON, M.; ROBERSON, D.; VAN DER VYER, J.; BARRET, L. Perceptions of emotion from facial expressions are not culturally universal: Evidence from a remote culture. Emotion, v. 14, n. 2, p. 251-262, 2014.

GOLEMAN, D. Inteligência emocional. Barcelona: Kairós, 1995.

HALBERSTAT, A. G.; LOZADA, F. T. Emotion development in infancy through the lens of culture. Emotion Review, v. 3, n. 2, p. 158-168, 2011.

HARMON-JONES, C.; SCHMEICHEL, B.; MENNITT, E.; HARMON-JONES, E. The expression of determination: Similarities between anger and approachrelated positive affect. Journal of Personality and Social Psychology, v. 100, n.1, p. 172-181, 2011.

HERDMAN, M.; FOX-RUSHBY J.; BADIA, X. Equivalence and the translation and adaptation of health-relates quality of life questionnaires. Qual Life Res, v. 6, p. 237-247, 1997.

HUMPHREY, N.; KALAMBOUKA, A.; WIGELSWORTH, M.; LENDRUM, A.; DEIGHTON, J.; WOLPERT, M. Measures of Social and Emotional Skills for Children and Young People: A Systematic Review. Educational and Psychological Measurement, v. 71, n.4, p.617-637, 2011.

IIZARD, C. E.; FINE, S.; SCHULTZ, D.; MOSTOW, A.; ACKERMAN, B.; YOUNGSTROM, E. Emotion Knowledge as a predictor of social behavior and academic competence in children at risk. Psychological Science, v. 12, n.1, 18-23. 2001.

IZARD, C. More Meanings and More Questions for the term "Emotion". Emotion Review, v. 2, n. 4, P. 383-385, 2010.

JACK, R. E.; GARROT, O. G.; YU, H.; CLADARA, R.; SCHYNS, P. G. Facial expressions of emotion are not culturally universal. Procedings of the National Academy of Sciences, v. 109, n. 19, p. 7241-7244, 2012.

JESUS, R. M.; LEMPKE, N. N. S. Manifestações emocionais das crianças na educação infantil. Synthesis Revista Digital FAPAM, v. 6, n. 6, p. 309-325, 2015.

JORGE, M. R. Adaptação transcultural de instrumentos de pesquisa em saúde mental. Revista Psiquiatria Clínica, v. 25, n. 15, p. 233-239, 1998. 
LINDQUIST, K.; SIEGEL, E.; QUIGLEY, K.; BARRET, L. The hundred-year emotion war: Are emotions natural kinds or psychological construction? Comment on Lench, Flores, and Bench. Psychological Bulletin, v. 139, n. 1, p. 255-263, 2013.

LOPATOCSKA, I.; ARAPAKIS, I. Theories, methods and current research on emotions in library and information science, information retrieval and humancomputer interaction. Information Processing \& Management, v. 47, n. 4, p. 575-592, 2011.

LÓPEZ CASSÁ, E. La educación emocional em la educación infantil. Revista interuniversitária de Formación del Profesorado, v. 16, n. 3, p. 153-167, 2005.

MACHADO, P.; VERÍSSIMO, M.; DENHAM, S. O teste de Conhecimento das Emoções para crianças de idade pré-escolar. Revista lberoamericana de Diagnóstico e Avaliação Psicológica, v. 1, n. 34, p. 201-222, 2012.

MACHADO, P.; VERÍSSIMO, M.; TORRES, N.; PECEGUINA, M. I. D.; SANTOS, A. J.; ROLÃO, T. Relações entre o conhecimento das emoções, as competências académicas, as competências sociais e a aceitação entre pares. Análise Psicológica, v. 26, n. 3, p. 463-478, 2008.

MARTINS, C. Manual de Análise de dados quantitativos com recurso ao IBM SPSS. Braga: Psiquilíbrios Edições, 2011.

MAYER, J. D.; SALOVEY, P. Que és Inteligencia Emocional? In J. M. Mestre e P. Fernández-Berrocal (eds.). Manual de Inteligência Emocional. Madrid: Pirâmide, p. 25-45, 2007.

MESTRE, J. M.; GUIL, R.; MARTINEZ-CABAÑAS, F.; LARRÁN, C.; GONZÁLEZ, G. Validación de uma prueba para evaluar la capacidade de percibir, expressar y valorar emociones em niños de la etapa infantil. Revista Electrónica Interuniversitária de Formación del Profesorado, v. 14, n. 3, p. 3754, 2011.

MOORS, A. On the casual role of appraisal in emotion. Emotion Review, 5, p. 356-368, 2013.

NOWICKI, S.; DUKE, M. P. Individual differences in the nonverbal communication of affect: the diagnostic analyses of nonverbal accuracy scale. Journal of Nonverbal Behavior, v. 18, n. 1, p. 9-35, 1994.

PETRIDES, K. V.; FURNHAM, A. On the dimensional structure of emotional intelligence. Personality and Individual Differences, v. 29, n. 2, p. 313-320, 2000.

PETRIDES, K. V.; VERNON, P.; SCHERMER, J.; VESELKA, L. Trait emotional intelligence and the dark traid traits of personality. Twin Research and Human Genetics, v. 14, n. 1, p. 35-41, 2011.

PLUTCHIK, R.; KELLERMAN, H. Theories of emotion. San Diego, C. A: Academic Press, 2013.

PLUTCHIK, R. Emotion: A psychoevolutionary synthesis. Harpercollins: College Division, 1980. 
RHOADES, B. L.; WARREN, H. K.; DOMITROVICH, C. E.; GREENBER, M. T. Examining the link between preschool social-emotional competence and first grade academic achievement: The role of attention skills. Early Childhood Research Quarterly, v. 26, n. 2, p. 182-191, 2011.

RICARTE, M. D.; MINERVINO, C. M.; ROAZZI, A.; DIAS, M. G. B.; VIANA, D. N. M. Crianças e suas emoções: análise da capacidade de reconhecimento das emoções (resumo). Trabalho apresentado no IV Congresso Brasileiro de Avaliação Psicológica, Campinas, Brasil, 2019.

RIVERS, S.; BRACKETT, M.; REYES, M.; MAYER, J.; CARUSO, D.; SALOVEY, P. Measuring emotional intelligence in early adolescence with the MSCEIT-IV: Psychometric properties and relations ship with academic performance and psychosocial functioning. Journal of Psychoeducational Assessment, v. 30, n. 4, p. 344-366, 2012.

RUSSO, P.; MANCINI, G.; TROMBINI, E.; BALDARO, B.; MAVROVELI, S.; PETRIDES, K. V. Trait emotional intelligence and he Big Five: A study on Italian children and preadolescents. Journal of Psychoeducational Assessment, v. 30, p. 274283, 2012.

SAARNI, C. Emotional development in childhood. Encyclopedia on Early Childhood Development, p.1-7, 2011.

SCHULTZ, D.; IZARD, C. E.; BEAR, G. Children's emotion processing: Relations to emotionality and aggression. Development and psychopathology, 16(2), 371388, 2004.

SHAFFER, D. R.; KIPP, K. Psicologia do desenvolvimento: infância e adolescência. São Paulo: Cengage Learning 2012.

SHERER, K. R.; EKMAN, P. Approaches to emotion. New York: Psychology Press, 2014.

VAN ZYL, C. The psychometric properties of the Emotional Quotient Inventory 2.0 in South Africa. Journal of Industrial Psychology, v. 40, n. 1, p.1-8, 2014.

\section{AGRADECIMENTOS}

A Jose Miguel Mestre e a todos os que direta ou indiretamente colaboraram neste estudo.

Recebido em: Setembro de 2017 Aprovado em: Março de 2018 